\title{
Suggestion for a new classification of earthquakes according to the focal depth
}

\author{
P. Heserviri $(*)$
}

Ricevuto il 25 . Marzo 1964

Sumary. - In this paper the Author suggests to regard the lower limit of the category of the so-called shallow earthquakes identical with the level of the regional isostatic compensation and the upper limit of the so-called deep shocks identical with the Byerly-discontinuity.

Riassuxro. - In questa nota l'A. sumgerisce di identificare il limite inferiore della categoria dei cosi detti terremoti a profondita normale con il livello della superficie di compensazione isostatica e il limite superiore dei così detti terremoti profondi con la discontinuità di Byerly.

In the geophysical practice it is customary to classify the earthquakes into three different groups according to the depth of hypocenter. In accordance with this classifying we distinguish shallow, intermediate and deep shocks respectively. According to the classical division:

Table I.

\begin{tabular}{|l|c|}
\hline \multicolumn{1}{|c|}{ Denomination } & Limits of focal depth, km \\
\cline { 2 - 2 } Shallow & $0-70$ (sometimes 0-60) \\
Intermediate & $70-300$ \\
Deep & $300-750$ \\
\hline
\end{tabular}

(*) F.R.A.S. Vice-Presilent of International Lunar Society. 
Some authors, e.g. Ritsema $\left({ }^{2}\right)$ and Honda $\left({ }^{3}\right)$ used a somewhat different classifying than that of in Table $I$. On the 6th figure of Ritsema's paper we find the following division of shocks:

Table II.

\begin{tabular}{|c|c|}
\hline Group & Limits of focal depth. km \\
\hline I. & $0-40$ \\
\hline II. & $40-130$ \\
\hline III. & $\geq 130$ \\
\hline
\end{tabular}

In his quoted paper Honda wrote the followings: "The earthquakes are classed here as shallow when the focal depth does not exceed $100 \mathrm{~km}$, intermediate when it is from $100 \mathrm{~km}$ to $250 \mathrm{~km}$, and deep when it exceeds $250 \mathrm{~km} "$.

There is no particular geophysical reason to maintain further the classical division of earthquakes (see in Table I). It seems to be more real if we comnect the limits of focal depth with certain special geophysical levels in the Earth. Such levels may be the different discontimuities; the Nohorovicic-surface, the Byerly- and Repetti-ones.

However here appears a rather great difficulty. As it is wellknown, the depth of the Nohorovicic-discontinuity is varying from place to place in accordance with the regional relief of the surface and the rule of isostasy. Although there are many data of the Earth's crustal thickness, due to gravitational and seismical explorations, nevertheless the number of useful values is as the present too small for a theoretical world-wide investigntion.

On the other hand the crustal thickness under oceanic territories is only $5-15 \mathrm{~km}$ or somewhat larger'. If we comnect the lower limit of the shallow shocks' eategory with the Mohorovicic-discontinuity, the greatest part of earthquakes over the oceanic territories (earlier pertaining to the shallow category), - would now belong into the group of the intermediate quakes. Tereby much of the regularities discovered concerning the shallow shocks all over the world would disappear. Natulally this were not desirable. Therefore we suggest to regard the lower limit of the shallow shocks' category idlentical with the level of the regional isostatic 
compensation. According to the calculations of Gutenberg $\left({ }^{4}\right)$ the pressure between the surface and the depth of $50 \mathrm{~km}$ is as follows (See on Table III):

Table III.

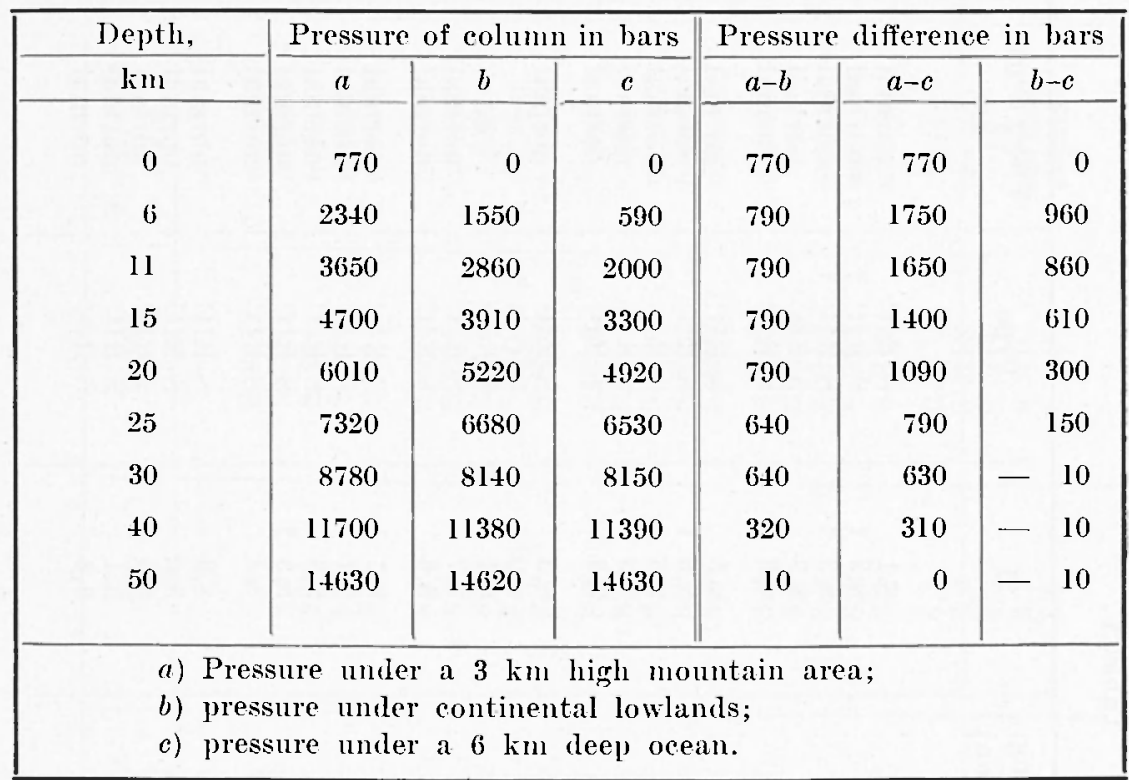

It is to be seen that the difference of pressure in a depth of $50 \mathrm{~km}$ is almost zero. Therefore we may regard the depth of $50 \mathrm{~km}$ as the level of regional isostatic compensation.

In accordance with these considerations the suggested new classification of earthquakes regarding their focal depth is as follows:

Table IV.

\begin{tabular}{|c|c|c|}
\hline Category & Limits of focal depth & $\mathrm{km}$ \\
\hline S-RIC & $\begin{array}{l}\text { Surface, - level of regional } \\
\text { isostatic compensation }\end{array}$ & $0-50$ \\
\hline $\mathrm{RIC}-\mathrm{B}$ & $\begin{array}{l}\text { Level of regrional isostatic } \\
\text { compensation, - Byerly-discon- } \\
\text { tinuity }\end{array}$ & $51-413$ \\
\hline $\mathrm{B}-\mathrm{R}$ & $\begin{array}{l}\text { Byerly-discontinuity, - } \\
\text { Repetti-discontinuity }\end{array}$ & $>414$ \\
\hline
\end{tabular}


Table V.

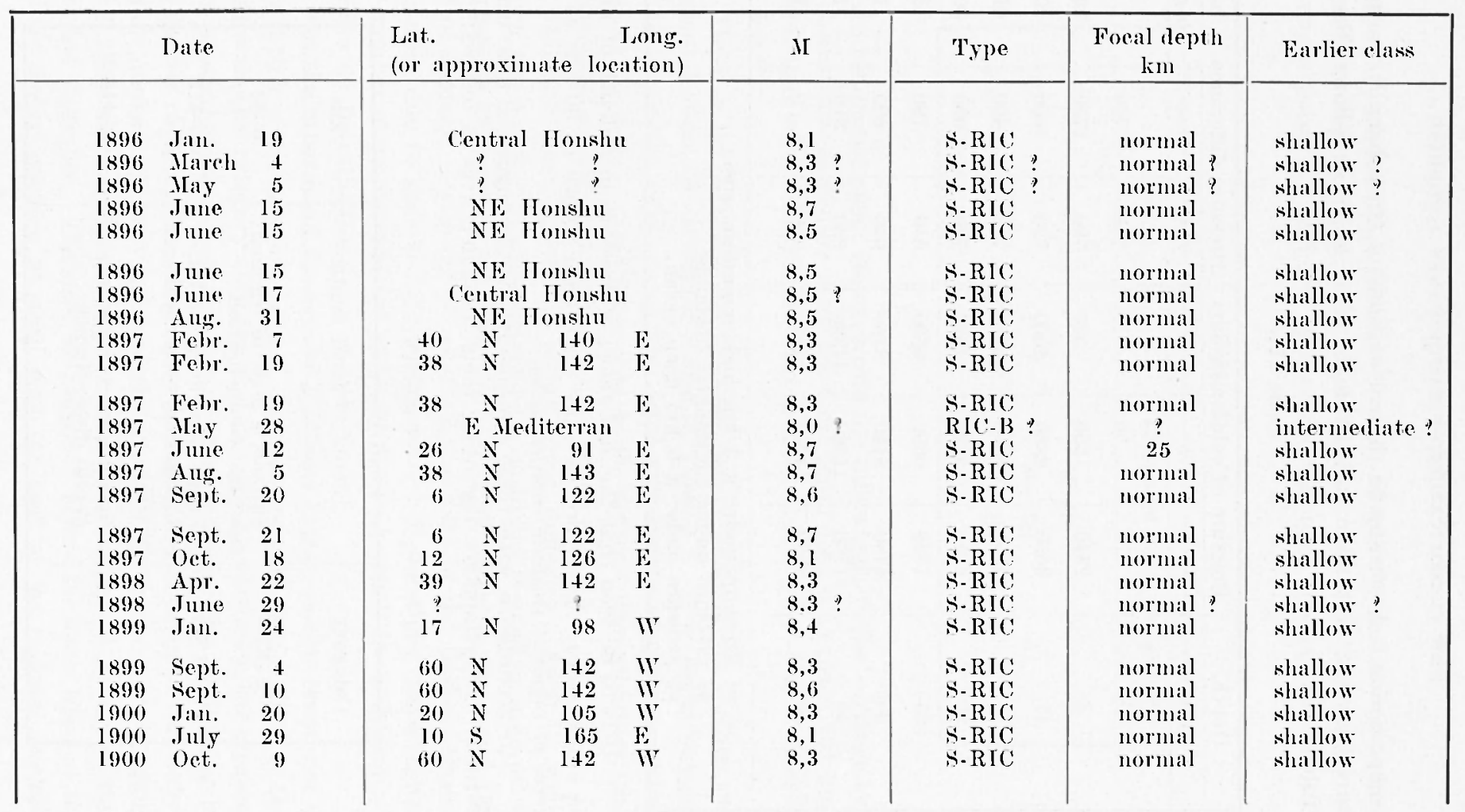


(Continuation)

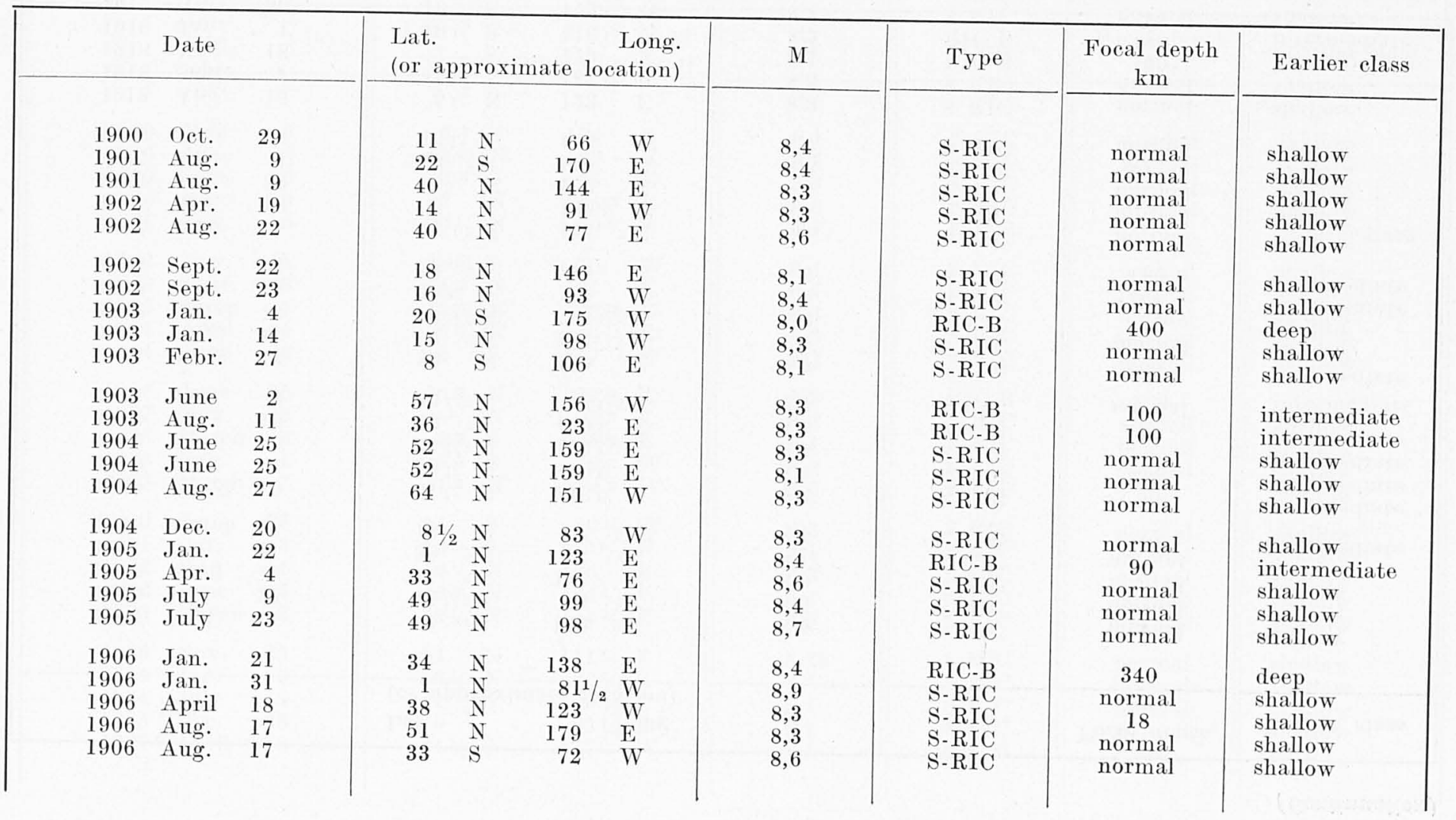


(Continuation)

\begin{tabular}{|c|c|c|c|c|c|c|c|c|}
\hline \multicolumn{3}{|c|}{ Date } & \multicolumn{2}{|c|}{$\begin{array}{l}\text { Lat. } \\
\text { (or approximate location) }\end{array}$} & $\begin{array}{l}\text { M } \\
8,4\end{array}$ & \multirow{2}{*}{$\begin{array}{l}\text { Type } \\
\\
\text { S-RIC } \\
\text { S-RIC } \\
\text { S-RIC } \\
\text { S-RIC } \\
\text { RIC-B }\end{array}$} & \multirow{2}{*}{$\begin{array}{c}\text { Focal depth } \\
\text { km } \\
\\
\text { normal } \\
\text { normal } \\
\text { normal } \\
\text { normal } \\
80\end{array}$} & \multirow{2}{*}{$\begin{array}{l}\text { Earlier class } \\
\text { shallow } \\
\text { shallow } \\
\text { shallow } \\
\text { shallow } \\
\text { intermediate }\end{array}$} \\
\hline $\begin{array}{l}1906 \\
1906 \\
1907 \\
1907 \\
1908\end{array}$ & $\begin{array}{l}\text { Sept. } \\
\text { Dec. } \\
\text { April } \\
\text { Oct. } \\
\text { March }\end{array}$ & $\begin{array}{l}14 \\
22 \\
15 \\
21 \\
26\end{array}$ & $\begin{array}{ll}7 & \mathrm{~S} \\
43^{1 / 2} & \mathrm{~N} \\
17 & \mathrm{~N} \\
38 & \mathrm{~N} \\
18 & \mathrm{~N}\end{array}$ & $\begin{array}{rl}149 & \mathrm{E} \\
85 & \mathrm{E} \\
100 & \mathrm{~W} \\
69 & \mathrm{E} \\
99 & \mathrm{~W}\end{array}$ & $\begin{array}{l}8,4 \\
8,3 \\
8,3 \\
8,1 \\
8,1\end{array}$ & & & \\
\hline $\begin{array}{l}1909 \\
1909 \\
1910 \\
1910 \\
1911\end{array}$ & $\begin{array}{l}\text { March } \\
\text { July } \\
\text { April } \\
\text { June } \\
\text { Jan. }\end{array}$ & $\begin{array}{r}13 \\
7 \\
12 \\
16 \\
3\end{array}$ & $\begin{array}{ll}311 / 2 & \mathrm{~N} \\
361 / 2 & \mathrm{~N} \\
251 / 2 & \mathrm{~N} \\
19 & \mathrm{~S} \\
431 / 2 & \mathrm{~N}\end{array}$ & $\begin{array}{rl}1421 / 2 & \mathrm{E} \\
701 / 2 & \mathrm{E} \\
1221 / 2 & \mathrm{E} \\
1691 / 2 & \mathrm{E} \\
771 / 2 & \mathrm{E}\end{array}$ & $\begin{array}{l}8,3 \\
8,1 \\
8,3 \\
8,6 \\
8,7\end{array}$ & $\begin{array}{l}\text { RIC-B } \\
\text { RIC-B } \\
\text { RIC-B } \\
\text { RIC-B } \\
\text { S-RIC }\end{array}$ & $\begin{array}{c}80 \\
230 \\
200 \\
100 \\
\text { normal }\end{array}$ & $\begin{array}{l}\text { intermediate } \\
\text { intermediate } \\
\text { intermediate } \\
\text { intermediate } \\
\text { shallow }\end{array}$ \\
\hline $\begin{array}{l}1911 \\
1911 \\
1913 \\
1913 \\
1914\end{array}$ & $\begin{array}{l}\text { June } \\
\text { Aug. } \\
\text { March } \\
\text { Oct. } \\
\text { Nov. }\end{array}$ & $\begin{array}{l}15 \\
16 \\
14 \\
14 \\
24\end{array}$ & $\begin{array}{ll}29 & \mathrm{~N} \\
7 & \mathrm{~N} \\
41 / 2 & \mathrm{~N} \\
191 / 2 & \mathrm{~S} \\
22 & \mathrm{~N}\end{array}$ & $\begin{array}{ll}129 & \mathrm{E} \\
137 & \mathrm{E} \\
1261 / 2 & \mathrm{E} \\
169 & \mathrm{E} \\
143 & \mathrm{E}\end{array}$ & $\begin{array}{l}8,7 \\
8,1 \\
8,3 \\
8,1 \\
8,7\end{array}$ & $\begin{array}{l}\text { RIC-B } \\
\text { S-RIC } \\
\text { S-RIC } \\
\text { RIC-B } \\
\text { RIC-B }\end{array}$ & $\begin{array}{c}160 \\
\text { normal } \\
40 \\
230 \\
110\end{array}$ & $\begin{array}{l}\text { intermediate } \\
\text { shallow } \\
\text { shallow } \\
\text { intermediate } \\
\text { intermediate }\end{array}$ \\
\hline $\begin{array}{l}1915 \\
1916 \\
1917 \\
1917 \\
1917\end{array}$ & $\begin{array}{l}\text { May } \\
\text { Jan. } \\
\text { Jan. } \\
\text { May } \\
\text { June }\end{array}$ & $\begin{array}{r}1 \\
13 \\
30 \\
1 \\
26\end{array}$ & $\begin{array}{ll}47 & \mathrm{~N} \\
3 & \mathrm{~S} \\
561 / 2 & \mathrm{~N} \\
29 & \mathrm{~S} \\
151 / 2 & \mathrm{~S}\end{array}$ & $\begin{array}{ll}155 & \mathrm{E} \\
1351 / 2 & \mathrm{E} \\
163 & \mathrm{E} \\
177 & \mathrm{~W} \\
173 & \mathrm{~W}\end{array}$ & $\begin{array}{l}8,1 \\
8,1 \\
8,1 \\
8,6 \\
8,7\end{array}$ & $\begin{array}{l}\text { S-RIC } \\
\text { S-RIC } \\
\text { S-RIC } \\
\text { RIC-B } \\
\text { S-RIC }\end{array}$ & $\begin{array}{c}\text { normal } \\
\text { normal } \\
\text { normal } \\
50-60 \\
\text { normal }\end{array}$ & $\begin{array}{l}\text { shallow } \\
\text { shallow } \\
\text { shallow } \\
\text { shallow } \\
\text { shallow }\end{array}$ \\
\hline $\begin{array}{l}1918 \\
1918 \\
1918 \\
1919 \\
1919\end{array}$ & $\begin{array}{l}\text { Aug. } \\
\text { Sept. } \\
\text { Nov. } \\
\text { Jan. } \\
\text { Apr. }\end{array}$ & $\begin{array}{r}15 \\
7 \\
18 \\
1 \\
30\end{array}$ & $\begin{array}{cl}51 / 2 & \mathrm{~N} \\
451 / 2 & \mathrm{~N} \\
7 & \mathrm{~S} \\
191 / 2 & \mathrm{~S} \\
19 & \mathrm{~S}\end{array}$ & $\begin{array}{ll}123 & \mathrm{E} \\
1511 / 2 & \mathrm{E} \\
129 & \mathrm{E} \\
1761 / 2 & \mathrm{~W} \\
1721 / 2 & \mathrm{~W}\end{array}$ & $\begin{array}{l}8,3 \\
8,3 \\
8,1 \\
8,3 \\
8,4\end{array}$ & $\begin{array}{l}\text { S-RIC } \\
\text { S-RIC } \\
\text { RIC-B } \\
\text { RIC-B } \\
\text { S-RIC }\end{array}$ & $\begin{array}{c}\text { normal } \\
\text { normal } \\
190 \\
180 \\
\text { normal }\end{array}$ & $\begin{array}{l}\text { shallow } \\
\text { shallow } \\
\text { intermediate } \\
\text { inrermediate } \\
\text { shallow }\end{array}$ \\
\hline
\end{tabular}


(Continuation)

\begin{tabular}{|c|c|c|c|c|c|c|c|c|}
\hline \multicolumn{3}{|c|}{ Date } & \multicolumn{2}{|c|}{$\begin{array}{l}\text { Lat. Long } \\
\text { (or approximate location) }\end{array}$} & \multirow{2}{*}{$\begin{array}{l}\text { M } \\
\\
\\
8,1 \\
8,3 \\
8,3 \\
8,6 \\
8,1\end{array}$} & \multirow{2}{*}{$\begin{array}{l}\text { Type } \\
\\
\text { S-RIC } \\
\text { S-RIC } \\
\text { S-RIC } \\
\text { S-RIC } \\
\text { RIC-B }\end{array}$} & \multirow{2}{*}{$\begin{array}{c}\text { Focal depth } \\
\text { km } \\
\\
\text { normal } \\
\text { normal } \\
\text { normal } \\
\text { normal } \\
215\end{array}$} & \multirow{2}{*}{$\begin{array}{l}\text { Earlier class } \\
\text { shallow } \\
\text { shallow } \\
\text { shallow } \\
\text { shallow } \\
\text { intermediate }\end{array}$} \\
\hline $\begin{array}{l}1919 \\
1910 \\
1920 \\
1920 \\
1921\end{array}$ & $\begin{array}{l}\text { May } \\
\text { June } \\
\text { Sept. } \\
\text { Dec. } \\
\text { Nov. }\end{array}$ & $\begin{array}{r}6 \\
5 \\
20 \\
16 \\
15\end{array}$ & $\begin{array}{ll}5 & \mathrm{~S} \\
231 / 2 & \mathrm{~N} \\
20 & \mathrm{~S} \\
36 & \mathrm{~N} \\
361 / 2 & \mathrm{~N}\end{array}$ & $\begin{array}{ll}154 & \mathrm{E} \\
122 & \mathrm{E} \\
168 & \mathrm{E} \\
105 & \mathrm{E} \\
701 / 2 & \mathrm{E}\end{array}$ & & & & \\
\hline $\begin{array}{l}1922 \\
1923 \\
1923 \\
1924 \\
1924\end{array}$ & $\begin{array}{l}\text { Nov. } \\
\text { Febr. } \\
\text { Sept. } \\
\text { April } \\
\text { June }\end{array}$ & $\begin{array}{r}11 \\
3 \\
1 \\
14 \\
26\end{array}$ & $\begin{array}{ll}281 / 2 & \mathrm{~S} \\
54 & \mathrm{~N} \\
351 / 4 & \mathrm{~N} \\
61 / 2 & \mathrm{~N} \\
56 & \mathrm{~S}\end{array}$ & $\begin{array}{ll}70 & W \\
161 & \mathrm{E} \\
1391 / 2 & \mathrm{E} \\
1261 / 2 & \mathrm{E} \\
157 & 1 / 2 \mathrm{E}\end{array}$ & $\begin{array}{l}8,4 \\
8,4 \\
8,3 \\
8,3 \\
8,3\end{array}$ & $\begin{array}{l}\text { S-RIC } \\
\text { S-RIC } \\
\text { S-RIC } \\
\text { S-RIC } \\
\text { S-RIC }\end{array}$ & $\begin{array}{l}\text { normal } \\
\text { normal } \\
\text { normal } \\
\text { normal } \\
\text { normal }\end{array}$ & $\begin{array}{l}\text { shallow } \\
\text { shallow } \\
\text { shallow } \\
\text { shallow } \\
\text { shallow }\end{array}$ \\
\hline $\begin{array}{l}1926 \\
1927 \\
1928 \\
1928 \\
1929\end{array}$ & $\begin{array}{l}\text { June } \\
\text { May } \\
\text { March } \\
\text { Dec. } \\
\text { March }\end{array}$ & $\begin{array}{r}26 \\
22 \\
9 \\
1 \\
7\end{array}$ & $\begin{array}{ll}361 / 2 & \mathrm{~N} \\
36^{3} / 4 & \mathrm{~N} \\
21 / 2 & \mathrm{~S} \\
35 & \mathrm{~S} \\
51 & \mathrm{~N}\end{array}$ & $\begin{array}{cl}271 / 2 & \mathrm{E} \\
102 & \mathrm{E} \\
881 / 2 & \mathrm{E} \\
72 & \mathrm{~W} \\
170 & \mathrm{~W}\end{array}$ & $\begin{array}{l}8,3 \\
8,3 \\
8,1 \\
8,3 \\
8,6\end{array}$ & $\begin{array}{l}\text { RIC-B } \\
\text { S-RIC } \\
\text { S-RIC } \\
\text { S-RIC } \\
\text { S-RIC }\end{array}$ & $\begin{array}{c}100 \\
\text { normal } \\
\text { normal } \\
\text { normal } \\
50\end{array}$ & $\begin{array}{l}\text { intermediate } \\
\text { shallow } \\
\text { shallow } \\
\text { shallow } \\
\text { shallow }\end{array}$ \\
\hline $\begin{array}{l}1929 \\
1931 \\
1932 \\
1932 \\
1933\end{array}$ & $\begin{array}{l}\text { June } \\
\text { Oct. } \\
\text { May } \\
\text { June } \\
\text { March }\end{array}$ & $\begin{array}{r}27 \\
3 \\
14 \\
3 \\
2\end{array}$ & $\begin{array}{rl}54 & \mathrm{~S} \\
101 / 2 & \mathrm{~S} \\
01 / 2 & \mathrm{~N} \\
191 / 2 & \mathrm{~N} \\
391 / 4 & \mathrm{~N}\end{array}$ & $\begin{array}{ll}291 / 2 & W \\
161^{3} / 4 & \mathrm{E} \\
126 & \mathrm{E} \\
1041 / 4 & W \\
1441 / 2 & \mathrm{E}\end{array}$ & $\begin{array}{l}8,3 \\
8,1 \\
8,3 \\
8,1 \\
8,9\end{array}$ & $\begin{array}{l}\text { S-RIC } \\
\text { S-RIC } \\
\text { S-RIC } \\
\text { S-RIC } \\
\text { S-RIC }\end{array}$ & $\begin{array}{l}\text { normal } \\
\text { normal } \\
\text { normal } \\
\text { normal } \\
\text { normal }\end{array}$ & $\begin{array}{l}\text { shallow } \\
\text { shallow } \\
\text { shallow } \\
\text { shallow } \\
\text { shallow }\end{array}$ \\
\hline $\begin{array}{l}1933 \\
1934 \\
1934 \\
1935 \\
1937\end{array}$ & $\begin{array}{l}\text { Nov. } \\
\text { Jan. } \\
\text { July } \\
\text { Dec. } \\
\text { April }\end{array}$ & $\begin{array}{l}25 \\
15 \\
18 \\
28 \\
16\end{array}$ & $\begin{array}{ll}34 & \mathrm{~N} \\
261 / 2 & \mathrm{~N} \\
11^{3 / 4} & \mathrm{~S} \\
0 & \\
211 / 2 & \mathrm{~S}\end{array}$ & $\begin{array}{rl}1411 / 2 & \mathrm{E} \\
861 / 2 & \mathrm{E} \\
1661 / 2 & \mathrm{E} \\
981 / 4 & \mathrm{E} \\
177 & \mathrm{~W}\end{array}$ & $\begin{array}{l}8,25 \\
8,4 \\
8,1 \\
8,1 \\
8,1\end{array}$ & $\begin{array}{l}\text { S-RIC } \\
\text { S-RIC } \\
\text { S-RIC } \\
\text { S-RIC } \\
\text { RIC-B }\end{array}$ & $\begin{array}{c}\text { normal } \\
\text { normal } \\
\text { normal } \\
\text { normal } \\
400\end{array}$ & $\begin{array}{l}\text { shallow } \\
\text { shallow } \\
\text { shallow } \\
\text { shallow } \\
\text { deep }\end{array}$ \\
\hline
\end{tabular}


(Continuation)

\begin{tabular}{|c|c|c|c|c|c|c|c|c|}
\hline \multicolumn{3}{|c|}{ Date } & \multicolumn{2}{|c|}{$\begin{array}{l}\text { Lat. } \\
\text { (or aplug. } \\
\text { roximate location) }\end{array}$} & $\begin{array}{c}\text { M } \\
8,6\end{array}$ & $\begin{array}{l}\text { Type } \\
\text { S.RIC }\end{array}$ & $\begin{array}{l}\begin{array}{c}\text { Focal depith } \\
\text { km }\end{array} \\
\text { nวma }\end{array}$ & \multirow{2}{*}{$\begin{array}{l}\text { Larlier class } \\
\\
\text { shalio's } \\
\text { shallo'y } \\
\text { shallow } \\
\text { shallo' } \\
\text { interne liate }\end{array}$} \\
\hline $\begin{array}{l}1938 \\
1938 \\
1939 \\
1939 \\
19939\end{array}$ & $\begin{array}{l}\text { Febr. } \\
\text { Nov. } \\
\text { Jan. } \\
\text { Ipril } \\
\text { Iec. }\end{array}$ & $\begin{array}{r}1 \\
10 \\
25 \\
30 \\
21\end{array}$ & $\begin{array}{rl}51 / 1 & \mathrm{~S} \\
551 \% & \mathrm{~N} \\
361 / 4 & \mathrm{~K} \\
101 / 2 & \mathrm{~S} \\
0 & \end{array}$ & $\begin{array}{cl}1301 / 2 & \mathrm{E} \\
158 & \mathrm{~W} \\
721 / 4 & \mathrm{~W} \\
1581 / 2 & \mathrm{E} \\
123 & \mathrm{E}\end{array}$ & $\begin{array}{l}8,6 \\
8.7 \\
8,3 \\
8,1 \\
8,6\end{array}$ & $\begin{array}{l}\text { S.RIC } \\
\text { A.RIC } \\
\text { RIC-B } \\
\text { S.RIC } \\
\text { PIC-IS }\end{array}$ & $\begin{array}{c}\text { nomai } \\
\text { normal } \\
5 \text { joc } \\
50 \\
150\end{array}$ & \\
\hline $\begin{array}{l}1940 \\
1941 \\
1941 \\
1942 \\
1942\end{array}$ & $\begin{array}{l}\text { May } \\
\text { June } \\
\text { Nov. } \\
\text { May } \\
\text { Aug. }\end{array}$ & $\begin{array}{r}24 \\
25 \\
25 \\
14 \\
6\end{array}$ & $\begin{array}{ccc}101 / 2 & S \\
121 / 2 & N \\
371 / 2 & N \\
0^{3} / 4 & \mathrm{~N} \\
14 & \mathrm{~N}\end{array}$ & $\begin{array}{ll}77 & \mathrm{VT} \\
92 \frac{1}{2} & \mathrm{E} \\
18^{1 / 2} & \mathrm{~W} \\
8 I^{1 / 2} & \mathrm{~W} \\
91 & \mathrm{~W}\end{array}$ & $\begin{array}{l}8,4 \\
8,7 \\
8,4 \\
8,3 \\
8,3\end{array}$ & $\begin{array}{l}\text { RIC-B } \\
\text { RIC }-B \\
\text { S-RIC } \\
\text { S.RIC } \\
\text { PIC } . B\end{array}$ & $\begin{array}{c}60 \\
60 \\
1 \text { orinal } \\
\text { no!roal } \\
50-60\end{array}$ & $\begin{array}{l}\text { sinallor } \\
\text { shallor } \\
\text { shallor } \\
\text { shallor } \\
\text { shallor }\end{array}$ \\
\hline $\begin{array}{l}1942 \\
1942 \\
1943 \\
1943 \\
1943\end{array}$ & $\begin{array}{l}\text { Aug. } \\
\text { Nov. } \\
\text { April } \\
\text { May } \\
\text { July }\end{array}$ & $\begin{array}{r}24 \\
10 \\
6 \\
25 \\
23\end{array}$ & $\begin{array}{ll}15 & \mathrm{~S} \\
491 / 2 & \mathrm{~S} \\
30^{3} / 4 & \mathrm{~S} \\
71 / 2 & \mathrm{~N} \\
91 / 2 & \mathrm{~S}\end{array}$ & $\begin{array}{rl}76 & W \\
32 & \mathrm{E} \\
72 & \mathrm{~W} \\
128 & \dot{\mathrm{L}} \\
110 & \mathrm{E}\end{array}$ & $\begin{array}{l}8,6 \\
8,3 \\
8,3 \\
8,1 \\
8,1\end{array}$ & $\begin{array}{l}\text { RIC-B } \\
\text { S-RIC; } \\
\text { RIC-B } \\
\text { S-RIC } \\
\text { RIC-B }\end{array}$ & $\begin{array}{c}69 \\
\text { norna: } \\
50-60 \\
\text { nol. } \\
90\end{array}$ & $\begin{array}{l}\text { slallow } \\
\text { slatiloy } \\
\text { shallow } \\
\text { shall w } \\
\text { interme liate }\end{array}$ \\
\hline $\begin{array}{l}1944 \\
19+5 \\
1946 \\
19+6 \\
1948\end{array}$ & $\begin{array}{l}\text { Dec } \\
\text { Nor. } \\
\text { Aug. } \\
\text { Ioce. } \\
\text { Janl. }\end{array}$ & $\begin{array}{r}7 \\
27 \\
4 \\
20 \\
24\end{array}$ & $\begin{array}{lll}33^{3} & \mathrm{~N} \\
24 & \mathrm{~N} \\
191 / 4 & \mathrm{~N} \\
321 / 2 & \mathrm{~N} \\
101 / 2 & \mathrm{~N}\end{array}$ & $\begin{array}{cc}136 & \mathrm{E} \\
63 & \mathrm{E} \\
69 & \mathrm{~W} \\
1341 / 2 & \mathrm{E} \\
122 & \mathrm{E}\end{array}$ & $\begin{array}{l}8,3 \\
8,3 \\
8,1 \\
8,4 \\
8,3\end{array}$ & $\begin{array}{l}\text { S-RIC } \\
\text { S-RIC! } \\
\text { S-RIC } \\
\text { S-RIC } \\
\text { S-RI }\end{array}$ & $\begin{array}{l}\text { normial } \\
\text { norrial } \\
\text { normal } \\
\text { norroal } \\
\text { normial }\end{array}$ & $\begin{array}{l}\text { shallow } \\
\text { shallow } \\
\text { shallow } \\
\text { shallow } \\
\text { shallow }\end{array}$ \\
\hline $\begin{array}{l}1949 \\
1950 \\
1950 \\
1950 \\
1950\end{array}$ & $\begin{array}{l}\text { Aug. } \\
\text { Aug. } \\
\text { Nov. } \\
\text { Dec. } \\
\text { l)ec. }\end{array}$ & $\begin{array}{r}22 \\
15 \\
2 \\
2 \\
9\end{array}$ & $\begin{array}{rl}53^{3} / 4 & \mathrm{~N} \\
281 / 2 & \mathrm{~N} \\
61 / 9 & N \\
181 / 4 & \mathrm{~S} \\
231 / 2 & \mathrm{~S}\end{array}$ & $\begin{array}{rl}1331 / 4 & \mathrm{~W} \\
961 \% 2 & \mathrm{E} \\
1291 / 2 & \mathrm{E} \\
1671 / 2 & \mathrm{E} \\
671 / 2 & \mathrm{~W}\end{array}$ & $\begin{array}{l}8,1 \\
8,7 \\
8,1 \\
8,1 \\
8,3\end{array}$ & $\begin{array}{l}\text { S-RIC } \\
\text { S-RIC } \\
\text { S-RIC } \\
\text { RIC-B } \\
\text { RIC-B }\end{array}$ & $\begin{array}{c}\text { norrial } \\
\text { llorilal } \\
50 \\
60 \\
100\end{array}$ & $\begin{array}{l}\text { shaliow } \\
\text { shalloy } \\
\text { sladiory } \\
\text { s'alloy } \\
\text { internediate }\end{array}$ \\
\hline
\end{tabular}


(Continuation)

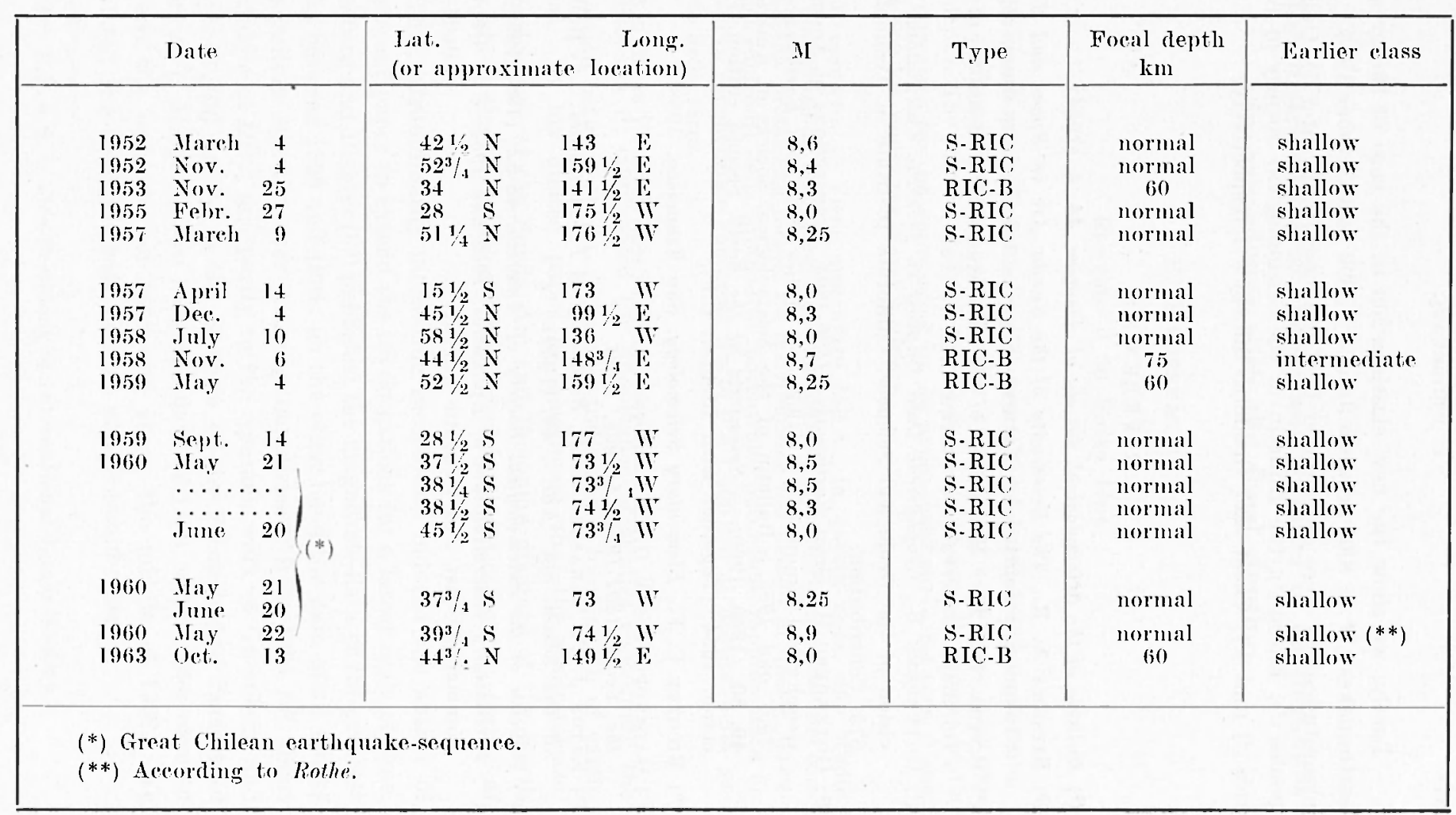


Lastly, we show the new classification in the case of the greatest earthquakes $(I I \geqslant 8,0)$ between January 1896 and October 1963 . The magnitude-data were determined by Gutenberg $\left(^{5}\right)$, Richter $\left({ }^{6}\right)$, Galanopoulos $\left({ }^{7}\right)$, Eaton $\left({ }^{8}\right)$, the Bulletin of the Seismologieal Society of America $\left({ }^{9}\right)$ and US Coast and Geodetic Survey $\left({ }^{10}\right)$ respectively.

\section{REFERENCES}

(1) Bulletin of the Seismological Society of America, 44, 4, (1954).

(2) Ritsena A. R., The Seismicity of the Sunda dre in space and Time. "Indonesian Journal for Natural seience ", 1-3, January-June, (1954).

(3) Hoxid II., The Mechanism of the Earthquakes. Publications of the Dominion Observatory, Ottawa, XX. 2, (1957).

(') Gutexnera: B.. Geophysical Data Implied in Isostalic Calculations. Division of the Geological Sciences, California Institute of Technology, 675. Contribution.

(5) Gutexierq B., Greal Earthquales 1896-1903. Transactions of American Geophysical Vnion. 37. 5, (1956). - Major and Great Earthquakes of 19.5 and 19.98. "Bulletin of the Seismological Soeiety of Ameriea", 49. 50. (1959, 1960). - Seismicity of the Earth. Second edition. Princeton. 1954. (Together with Richter, C.F).

$\left({ }^{6}\right)$ Ricurer C. F., Elementary Seismology. San Franciseo, 1958.

(i) Gatasopoulos A. G.. A Catalogue of Shocks with $I 0 \geqslant T I$ or $I I \geqslant 5$ for the Fears 1801-19.58. Athens, 1960.

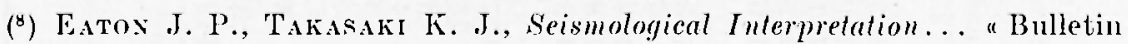
of the Seism. Soc. Am." 49. (1959).

$\left({ }^{9}\right)$ Bulletin of the Seismological Society of America. 43-52. (1953-1962).

(10) Preliminary Determinations of Epicentres, 1962 and 1963. V.S. Dept. of Commerce Coast and Geodetic Survey. 